Tecnología e Innovación

Tecnologia e Inovação

Tecnology and Innovation

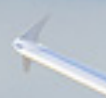

\title{
ESTRUCTURA DEL SOFTWARE: SISTEMA INTEGRADO DE OPERACIONES AÉREAS*
}

ESTRUTURA DE SOFTWARE: SISTEMA INTEGRADO DE OPERAÇÕES AÉREAS

Software Structure: Integrated System of Air Operations ${ }^{* * *}$

Sebastián Arroyave Cardona a Jaime Alejandro Lobo Zuluagab , German Alberto Barragán De Los Ríosc, German Urrea Quirogad y Jorge Iván García Sepúlvedae

Universidad Pontificia Bolivariana. Medellín, Colombia

\section{CIENCIA Y PODER AÉREO}

ISSN 1909-7050 / E- ISSN 2389-9468 / Volumen 12/ Enero-diciembre de 2017/ Colombia/ Pp. 210-219

Recibido: 03/09/2017

Aprobado: 02/10/2017

Doi: http://dx.doi.org/10.18667/cienciaypoderaereo.573 


\section{Para citar este artículo:}

Arroyave, S., Lobo, J. A., Barragán, G. A., Urrea, G., García, J. I. (2017). Estructura del software: sistema integrado de operaciones aéreas. Ciencia y Poder Aéreo, 12, 210219. Doi: http://dx.doi.org/10.18667/cienciaypoderaereo. 573

\section{'Artículo de investigación original \\ ${ }^{2}$ Artigo de pesquisa original}

${ }^{3}$ Original research article

a Ingeniero Aeronáutico, Universidad Pontificia Bolivariana, Gerente Comercial, Anse Electronics. Medellin, Colombia. Correo electrónico: arroyo45@ hotmail.com

b Ingeniero Aeronáutico, Universidad Pontificia Bolivariana, líder de confiabilidad, Aerolínea de Antioquia. Medellin, Colombia. Correo electrónico: lobozulu84@gmail.com

'Msc en Transporte Aéreo y Aeropuertos del Instituto Tecnológico de Aeronáutica, Ing. Aeronáutico de la Universidad de San Buenaventura. Profesor asociado Facultad de Ingeniería Aeronáutica Universidad Pontificia Bolivariana. Medellín, Colombia. Correo electrónico: german.barragan@upb.edu.co

${ }^{d}$ Msc en Administración de la Universidad Pontifica Bolivariana, Ingeniero Aeronáutico de la Universidad de San Buenaventura, Profesor Asociado Facultad de Ingeniería Aeronáutica Universidad Pontificia Bolivariana. Medellín, Colombia. Correo electrónico: german.urrea@upb.edu.co

e Msc en Thermal Power de Cranfield University, Ingeniero Aeronáutico de la Universidad de San Buenaventura, Profesor Asociado Facultad de Ingeniería Aeronáutica Universidad Pontificia Bolivariana. Medellín, Colombia. Correo electrónico: jorge. garcia@upb.edu.co
Resumen: el siguiente documento tiene como objetivo presentar un método de automatización de los procesos en el área de operaciones de empresas de transporte aéreo, los cuales se realizan de forma manual generando malas decisiones o retrasos en la toma de decisiones en organizaciones en crecimiento y con bajo presupuesto.

Automatizar procesos como los que se realizan en el área de control vuelos o programación de tripulaciones, ayuda a realizar de forma más eficiente la labor del personal encargado y así reducir los tiempos de programación de aeronaves y tripulaciones.

La comunicación es un factor clave en cualquier empresa para poder funcionar eficientemente, especialmente en una aerolínea, ya que esta depende de la sincronización entre diversas áreas como mantenimiento y operaciones, es por esta que las herramientas tecnológicas son de gran utilidad para mejorar la comunicación y los tiempos de respuesta entre las áreas. Para el cumplimiento del objetivo propuesto se empleó un proceso de investigación descriptiva y proyectiva.

Palabras clave: operaciones aéreas, Software, Control de vuelos, Gestión de operaciones

Resumo: O seguinte documento tem como objetivo apresentar um método de automação de processos na área de operações das empresas de transporte aéreo, que são realizadas manualmente gerando decisões precárias ou atrasos na tomada de decisões em organizações em crescimento com baixo orçamento .

Automatize processos como os realizados na área de controle de voo ou planejamento de tripulação, ajudam a realizar o trabalho do pessoal responsável de forma mais eficiente e, assim, reduzem os tempos de programação de aeronaves e tripulações.

A comunicação é um fator chave em qualquer empresa para operar de forma eficiente, especialmente em uma companhia aérea, uma vez que depende da sincronização entre várias áreas, como manutenção e operações, é por isso que as ferramentas tecnológicas são muito úteis para melhorar a comunicação e os tempos de resposta entre as áreas.

Para cumprir o objetivo proposto, foi utilizado um processo de pesquisa descritiva e projetiva.

Palavras-chave: Operações aéreas, Software, Controle de vôo, Gerenciamento de operações.

Abstract: The following document presents a method of automation of the processes in the operations department of air transport companies, which are carried out manually producing wrong decisions or delays in decision making, mainly at growing organizations and with low budget.

Automating processes such as those performed in the flight operations or scheduling of crew's department, helps to perform the work of the personnel in charge more efficiently and thus reduce assigned times for aircraft and crews.

Communication is a key factor in any company to be able to operate efficiently, especially in an airline, where it depends on the synchronization between different departments such as maintenance and operations, it is for this reason that technological tools are very useful to improve communication and response times between departments.

To fulfill the proposed objective, a descriptive and projective research process was used.

Key Words: Air Operations, Software, Flight Control, Operations Management. 


\section{Glosario}

ARR: Arrival. Hora de llegada programada [horas, minutos] AM o PM.

Base: lugar donde opera la aerolínea que cuenta con personal de la empresa donde se embarcan y/o desembarcan pasajeros, correo o carga.

Itinerario: configuración de una o varias rutas para determinar el recorrido completo de un día de servicio.

MySQL: sistema de gestión de bases de datos (SGBD) multiusuario, multiplataforma y de código abierto.

Programación diaria: asignación de jornadas, itinerarios, aeronaves y tripulantes para cada día del mes.

RAC: Reglamentos Aeronáuticos de Colombia.

Ruta: dos o más trayectorias donde la ciudad origen de la primera trayectoria debe ser la ciudad destino de la última trayectoria.

SQL: Structured Query Language, lenguaje de consulta estructurado. Es un lenguaje surgido de un proyecto de investigación de IBM para el acceso a bases de datos relacionales. Actualmente se ha convertido en un estándar de lenguaje de bases de datos, y la mayoría de los sistemas de bases de datos lo soportan, desde sistemas para ordenadores personales, hasta grandes ordenadores.

\section{Introducción}

La industria aérea es un sector diverso que requiere el apoyo de una gama variada de negocios auxiliares tales como mantenimiento, catering y agencias de viajes para llevar a cabo sus actividades. Los sistemas de transporte son elementos esenciales para el desarrollo de cualquier actividad económica, pues el transporte aéreo se considera una de las industrias más grandes del mundo y uno de los servicios más importantes de la economía colombiana. (Olivera, Cabrera, Bermúdez, \& Hernández, 2011)

Debido a su topografía, Colombia presenta grandes retos para la operación de los medios de transporte de superficie, lo cual le da al trasporte aéreo una posición importante como ente facilitador de la integración y la conectividad. En los últimos años, el crecimiento del transporte aéreo se ha acelerado, lo cual se ha acompañado desde la década del 90 por políticas públicas de liberalización del espacio aéreo en los mercados interno y externo. La competencia entre las empresas que prestan servicios de transporte aéreo comercial las ha obligado a desarrollar constantemente nuevas formas de aumentar su eficiencia, con el fin de incrementar su margen de utilidades mediante el mejoramiento de las áreas operacionales, logísticas, administrativas y de mantenimiento aeronáutico (Álvarez \& García, 2010).

Un factor clave para cualquier organización es el proceso de toma de decisiones basado en una adecuada administración de la información. El aprovechamiento de los recursos que la tecnología ofrece y la manera en que estos son utilizados por las organizaciones se convierte en un elemento diferenciador, el cual tiene como fin el proveer información clara, precisa y confiable. La toma de decisiones es una actividad crítica dentro de las organizaciones; de ello depende en gran medida el éxito que la organización pueda alcanzar en un momento dado (Arteaga, 2004; Cohen \& Asin, 2001).

\section{Estado del arte}

Actualmente, en el mercado se encuentran disponibles soluciones informáticas con un fuerte potencial de implementación por la industria aeronáutica colombiana, tales como ICARUS PLUS, SOMA ONLINE, VOLARTEC, SIO y SAP, entre otras. Sin embargo, muchas de estas aplicaciones no son de fácil adquisición para las pequeñas y medianas empresas de la industria de aviación colombiana debido a los altos costos para su compra y mantenimiento.

A pesar de existir acuerdos internacionales y documentos técnicos que otorgan los lineamientos principales para la operación de las aeronaves a nivel mundial, la reglamentación (horas de vuelo, horas de servicio, tiempo de descanso, entre otros) cambia de país a país. Por esta razón, se hace necesario acoplar las soluciones a las reglamentaciones impuestas por la Uaeac (Unidad Administrativa Especial de Aeronáutica Civil), que para el caso colombiano es la autoridad con jurisdicción sobre la aviación civil.

Con el auge de la informática, en los últimos años se han desarrollado aplicaciones de muy buena calidad pero que no alcanzan a suplir todas las necesidades de la industria de aviación nacional, bien sea por precio, funcionalidad, o una combinación de ambas. A continuación, se presentará una rápida descripción de las principales soluciones informáticas que están disponibles en el mercado:

ICARO PLUS (Seicotechnology, 2014): integra tres grandes áreas de las compañías aéreas: área técnica, inventarios y operaciones. Cada una de ellas toma en cuenta lo siguiente:

- Área técnica: ingeniería, programación, planeación, control producción, mantenimiento y control de calidad. 
- Inventarios: componentes reparables, materiales, herramientas, valorización, cierres contables, garantías, centros de costo, requisiciones, órdenes de compra, stock mínimo, límites de almacenamiento.

- Operaciones: asignación de tripulantes, reportes a la autoridad aeronáutica, estadística de tripulaciones, control de chequeos, manejo de carga, consumos de combustible, reportes estadísticos por aeronave, flota y tripulantes.

SOMA (Solutions, 2014): Software de Operaciones y Mantenimiento Aeronáutico. Ayuda a controlar y rentabilizar las operaciones, mantenimiento y administración de talleres de servicio, taxi aéreo, aerolínea comercial o de carga, o cualquier otro tipo de empresa que opere aeronaves de ala fija o rotatoria.

En adición a los anteriores, se encontró y analizó otro tipo de software que tiene aplicaciones industriales importantes en diferentes aéreas, pero sin aplicaciones aeronáuticas directas.

\section{Problemática encontrada}

En un mercado altamente competitivo y dinámico, los operadores aéreos se encuentran obligados a optimizar sus costos, incrementar la eficiencia de sus operaciones y aumentar la calidad de sus servicios. Actualmente, la industria se caracteriza por presentar reducidos márgenes de rentabilidad, siendo el control y medición de los procesos los mejores caminos para hacer frente a este desafío (VolarTec, 2014).

El área de operaciones de una empresa aérea se considera uno de los entes principales y con mayor criticidad al tener relación directa con la programación de vuelos, tripulaciones, entrenamientos y equipos, entre otros. Cualquier falla en esta área podría generar un colapso general en el funcionamiento de la compañía, el cual conllevaría a retrasos y cancelaciones de vuelo que desembocarían en un fuerte lucro cesante, lo cual se traduce en la pérdida económica de una empresa por dejar de tener utilidad o ganancia, sumado a los gastos en que se puede incurrir por compensaciones económicas y sanciones.

Para el funcionamiento normal del área de operaciones se maneja una gran cantidad de información. Muchas organizaciones aeronáuticas utilizan hojas de cálculo para registrar, analizar y reportar las diferentes actividades relacionadas con el área, así como las tareas ejecutadas. Por ello se hace muy complejo el proceso de consolidación de información para generar reportes integrados de la empre- sa, así como solicitudes de cumplimiento por parte de la autoridad aeronáutica.

Durante el desarrollo de la investigación se identificó que existen necesidades específicas y concretas en las empresas de transporte aéreo comercial secundario de carga y pasajeros, respecto a disminuir los gastos y el tiempo en las tareas que se llevan a cabo en el área de operaciones. Esto, sumado a las condiciones actuales del mercado, genera una presión cada vez mayor sobre las compañías aéreas frente a la reducción de costos operacionales, lo cual redunda en la necesidad de realizar una optimización de los procesos asociados con la gerencia de operaciones a través de un sistema de información. Se esperaría que eso permita llevar a cabo las actividades de los procesos de esta área (control de tripulaciones, control de aeronaves y estadísticas) de una forma más rápida, con bajos niveles de fallos y en un ambiente amigable para el usuario, en el cual predominen funciones automáticas alrededor de los procesos, todo ello a un menor costo.

\section{Solución propuesta}

El software propuesto se denomina SIO (Sistema Integrado de Operaciones). Está dividido en cinco módulos que controlan toda el área de operaciones: control mensual de tripulación, control diario de tripulación, generación de estadísticas, control de aeronaves y control de operaciones.

Cada módulo administra y facilita las labores del personal del área encargada y, en conjunto, optimizan todo el proceso de la aerolínea. La base de datos será construida bajo el motor MySQL, el cual permite generar: las estructuras de datos de la base, las restricciones de integridad y las operaciones de manipulación de los datos.

El diseño de la base de datos del sistema está dado por un modelo entidad-relación, a través del cual se permite identificar las diferentes entidades del sistema de información y las relaciones existentes entre ellas. Adicionalmente, se orienta el modelo bajo el esquema relacional, con lo cual se logra un alto nivel de normalización.

La programación del sistema de información SIO se dio en una estructura por capas que tiene como objetivo primordial la separación de la lógica de negocios y de diseño (interfaces gráficas). Una de las consideraciones más importantes es independizar (desacoplar) la lógica de acceso a datos de la capa de presentación de usuario. Utilizando este estilo, el desarrollo se lleva a cabo en varios niveles y, en caso de que sobrevenga algún cambio, sólo se ataca al nivel requerido sin tener que revisar entre código mezclado (alta mantenibilidad). Igualmente, se confía una misión 
simple a cada una de las capas, lo que permite el diseño de arquitecturas escalables, es decir, que pueden ampliarse con facilidad en caso de que las necesidades aumenten.

El diseño arquitectónico utilizado está dado por la representación en tres niveles o capas:

- Capa de presentación: también denominada capa de usuario, presenta el sistema al usuario, le comunica la información y captura la información en un mínimo de proceso (realiza un filtrado previo para comprobar que no hay errores de formato). También se conoce como interfaz gráfica y debe tener la característica de ser amigable, es decir, entendible y fácil de usar. Esta capa se comunica únicamente con la capa de modelo.

- Capa de modelo: es donde residen los programas que se ejecutan, se reciben las peticiones del usuario y se envían las respuestas tras el proceso. Se denomina capa de modelo o de negocio (e incluso de lógica del negocio) porque es aquí donde se establecen todas las reglas que deben cumplirse. Esta capa se comunica con la capa de presentación para recibir las solicitudes y presentar los resultados, y con la capa de datos para solicitar al gestor de base de datos almacenar o recuperar datos de él.

- Capa de datos: es donde residen los datos y es la encargada de acceder a los mismos. Está formada por uno o más gestores de bases de datos que realizan todo el almacenamiento de datos, reciben solicitudes de almacenamiento o recuperación de información desde la capa de modelo.

- Los módulos que constituyen el sistema SIO se explica a continuación:

\section{Control mensual de tripulación}

Al automatizar este proceso se consigue, en primera instancia, disminuir el tiempo en la elaboración de la programación mensual de tripulantes, ya que el sistema ofrece una propuesta de programación automática que tiene en cuenta los tiempos de vuelo, servicio, vencimiento de entrenamientos, simulador y chequeos, lo que normalmente demanda mucho tiempo para un programador de tripulaciones al tener que verificar estos tiempos por tripulante individualmente. Adicionalmente, al generar la programación mensual, el programa brinda la posibilidad de enviar una notificación por correo electrónico a todos los pilotos para que estén enterados de su asignación.
Para el programador de escuelas y entrenamientos es bastante tedioso tener que estar verificando por cada uno de los pilotos cuándo se le vence alguna novedad técnica, ya sea simulador, chequeo o escuela. Al implementarse, el programa genera alertas de los vencimientos de cualquier novedad técnica de los pilotos, de manera que dicho proceso se facilita para el programador.

El proceso de control mensual de tripulación realiza las siguientes funciones para cada tripulante: ejecutar asignación días libres automáticamente; dividir tripulantes en grupos de forma aleatoria para generar la programación sin que se excedan los límites de tiempo establecidos por la normatividad aeronáutica colombiana; identificar semanas del mes de programación para distribuir los grupos equitativamente; asignar días libres por tripulante de manera automática para todo el mes de programación; asignar días libres sugeridos por cada tripulante si alguno tiene una necesidad puntual; asignar días libres por tripulante manualmente si es requerido por el personal de control de tripulación; asignar días blancos por cada tripulante en caso de que los tiempos de servicio no cumplan los requisitos legales; identificar novedades técnicas próximas a vencerse; asignar novedades técnicas por cada tripulante; asignar días de servicio por cada tripulante de forma automática; asignar días de servicio por cada tripulante de modo manual; almacenar la programación mensual y aprobar programación mensual de los vuelos.

\section{Control diario de tripulación y Control de aeronaves}

Inicialmente, el programador de aeronaves y tripulantes debe tomar una lista de rutas que se deben cumplir diariamente y agruparlas para formar un itinerario, el cual se cumple de acuerdo al tipo de aeronave.

Luego de ordenar los itinerarios, debe asignarlos a las aeronaves teniendo en cuenta también que se encuentren disponibles para vuelo. Finalmente, de acuerdo a la programación mensual debe verificar cuáles pilotos se encuentran programados para vuelo, así como que cumplan con las horas máximas de vuelo, servicio, descanso, número de aterrizajes, escuelas, chequeos, simulador, entre otros. Para poder realizar este proceso, debe separar un largo tiempo de su jornada laboral.

Los resultados obtenidos en la implementación del programa son los siguientes:

El programa ofrece una propuesta automática de asignación de itinerarios para las aeronaves con sus respectivos tripulantes y valida que los itinerarios correspondan al tipo de 
aeronave, que la aeronave se encuentre disponible para vuelo, y que la tripulación cumpla con los diferentes requisitos.

Luego de esta propuesta, el programador puede modificarla de acuerdo a sus necesidades específicas: puede, por ejemplo, eliminar un itinerario a una aeronave y asignarle otro de la lista de itinerarios cargados en la base de datos. También puede agregar o eliminar rutas del itinerario o modificar su horario. Si hay inconsistencias en las modificaciones que realice el usuario, el sistema genera alertas indicándolas. Puede modificar tripulaciones en cualquier ruta del día y asignarle novedades técnicas como chequeos de ruta. Además, existe una interfaz que permite al personal de mantenimiento separar una aeronave para un servicio técnico en cualquier momento del día; de esa manera, el programador está informado de si dispone o no de la aeronave.

Al hacer uso del sistema, el programador de aeronaves puede realizar de forma más eficaz su labor, con lo cual disminuye los tiempos de elaboración de la programación y los posibles errores humanos que puede cometer al tener que validar tanta información.

En el área de control diario se administran las siguientes tareas: identificar tripulantes asignados a la fecha de programación en el día de operación; asignar tripulantes a cada itinerario diario de modo que todas las rutas del día tengan la tripulación asignada; identificar en cuál jornada de vuelo están programados los tripulantes asignados; calcular horas de vuelo acumuladas para cada tripulante e identificar si el tripulante es apto o no de acuerdo a las normas establecidas por la Aerocivil; identificar y programar las asignaciones de novedades técnicas por tripulante; por último, almacenar la programación diaria de tripulantes.

El módulo de control de aeronaves se ejecuta en el siguiente orden: obtener las aeronaves que están disponibles para programación diaria; cargar y seleccionar los itinerarios a programar; asignar manual y automáticamente los itinerarios a las aeronaves; asignar rutas de forma individual a aeronaves; finalmente, y de ser necesario, modificar los tiempos de las trayectorias.

\section{Generación de estadística}

Para generar las estadísticas de interés para el área comercial de una compañía, se hace necesario recolectar toda la información que normalmente es almacenada en diferentes tablas de Excel, y luego agruparlas para mostrar gráficas y datos. Esto demanda tiempo para el personal de estadísticas, el cual puede usarse mejor en el análisis de dichos datos.
El software SIO captura automáticamente todos estos datos y genera las tablas y gráficos de interés, como el número de pasajeros transportados por ruta, estadística de vuelos demorados y estadística de consumo de combustible. Al obtener estos datos de forma automática, se puede emplear más tiempo en análisis de estos datos, lo cual se considera de mayor importancia.

Para obtener información de la primera trayectoria de cada día del mes, se consultan los siguientes datos de la primera trayectoria de cada fecha del mes de generación de la estadística: fecha de operación, matrícula de la aeronave, origen, destino, nombre del piloto, hora de despegue, hora de rodaje, hora de aterrizaje, hora de parqueo, combustible antes del despegue, combustible al aterrizar, peso al despegue. Además, se consulta el valor del flujo de combustible (ff) para el tipo de aeronave según la matrícula de la aeronave de cada trayectoria consultada [lbs/min]. Finalmente, se agrupa la información obtenida por cada aeronave y trayectoria.

Para la estadística de consumo de combustible se aplicará la fórmula del factor de consumo por avión, por trayecto y a la misma hora. Lo anterior con el objetivo de tomar en cuenta cada uno de los factores que influyen en el consumo de combustible de una aeronave.

Los factores más importantes a tener en cuenta para el consumo de combustible de una aeronave son la temperatura, el nivel de vuelo, el peso de la aeronave, vientos en ruta, condición aerodinámica de la aeronave en particular (es decir, todo lo que pueda generar resistencia parásita e inducida en la aeronave) y el set de potencia que usen los pilotos para cada vuelo.

Si se hace el cálculo para el mismo trayecto, a la misma hora y con la misma aeronave, se puede hacer una buena aproximación de cuánto están consumiendo cada uno de los aviones. Dado que la temperatura va a ser aproximadamente igual y que el nivel de vuelo va a ser siempre el mismo, se tomarán los datos para una misma aeronave.

El factor de consumo puede ayudar a identificar qué piloto está consumiendo más o menos combustible, además de si el factor de consumo está por encima de la media aritmética más desviación estándar en repetidas ocasiones durante el mes para el mismo piloto. De ese modo, la empresa puede entrar a evaluar si dicho tripulante está siguiendo las técnicas de vuelo o los procedimientos, con lo cual logra hacer un seguimiento con el objetivo de controlar el consumo en cada vuelo. 
Los datos serán tomados del libro de vuelo de la aeronave, en los cuales se registra el tiempo de vuelo y el consumo de combustible acorde a este tiempo (combustible total usado). El combustible total usado se toma desde que la aeronave comienza a rodar hasta que parquea; no obstante, para el cálculo del factor de consumo se usará el combustible consumido desde que la aeronave despega hasta que parquea (combustible consumido). Se toma entonces el tiempo de carreteo y se multiplica por el flujo de combustible promedio de los dos motores, lo cual arroja como resultado el combustible consumido en tierra o combustible de carreteo. Al restar éste al combustible total usado se obtiene el combustible consumido, con el cual se calcula el factor de consumo a través de la siguiente ecuación:

Factor de Consumo $=\frac{\text { Combustible Consumido }[\mathrm{lbs}]}{\text { Tiempo TO to } L D G[\mathrm{~min}]} \times \frac{1}{\text { Peso al despegue }[\mathrm{lbs}]}$

- Tiempo TO to $L D G$ : tiempo que toma la aeronave desde que despega hasta que aterriza.

- Peso al despegue: peso en libras que posee la aeronave al momento del despegue.

- Combustible consumido: combustible usado desde que la aeronave despega hasta que aterriza.

Para realizar el cálculo del combustible consumido se sigue la siguiente ecuación:

$$
F C[\mathrm{lb}]=T U F-(F F \times T T)-(F F \times T T D)
$$

TUF: combustible total usado. Es el combustible usado desde que la aeronave comienza el rodaje hasta que parquea [lb].

$F F$ : flujo de combustible que consumen ambos motores de la aeronave por minuto, en mínimos (potencia necesaria para efectuar carreteo) [Lb/min].

$T T$ : tiempo de carreteo. Tiempo que toma la aeronave en desplazarse desde la plataforma hasta la cabecera de la pista y viceversa [min].

TTD: tiempo de carreteo en el destino [min].

Para calcular el valor del tiempo de carreteo en el origen de cada trayectoria consultada, se usa la siguiente fórmula:

Hora de despegue - Hora de rodaje [min]

Para calcular el valor del tiempo de carreteo en el destino de cada trayectoria consultada, se usa la siguiente fórmula:

Hora de parqueo - Hora de aterrizaje [min]
Para calcular el valor del combustible total usado de cada trayectoria consultada, se usa la siguiente fórmula:

Combustible antes del despegue - Combustible al aterrizar [lbs]

Para calcular el valor del tiempo TO to LDG de cada trayectoria consultada, se usa la siguiente fórmula:

\section{Hora de aterrizaje - Hora de despegue [min]}

Calcular valor límite del factor de consumo: se calcula el valor límite superior del factor de consumo de cada trayectoria (primera trayectoria de cada día del mes), consultada para el mes de generación de la estadística de consumo. Para esto se debe:

- Calcular el valor de la media aritmética de los factores de consumo calculados, agrupando dichos valores por aeronave y trayectoria.

- Calcular el valor de la desviación estándar de los factores de consumo calculados, agrupando dichos valores por aeronave y trayectoria.

- Calcular el valor del límite superior de los factores de consumo calculados con base en la siguiente fórmula:

\section{Media aritmética + Desviación estándar}

Dado que para estudiar los valores del factor de consumo por aeronave y trayectoria no es suficiente con un parámetro de centralización (media aritmética), es necesario obtener adicionalmente un parámetro de dispersión que indique si los datos estudiados están más concentrados o más dispersos (desviación estándar).

Debe tenerse en cuenta que los vuelos que tengan problemas o situaciones fuera de lo común y que afecten considerablemente el consumo de combustible de la aeronave deben descartarse manualmente de los datos estadísticos, de manera tal que no afecten la tendencia ni el desvío (la media aritmética y el desvío estándar se ven muy afectados por este tipo de datos). Con la media aritmética puede calcularse la desviación estándar, la cual es el filtro para saber qué datos se reportan como elevados. Los datos que superen el promedio más la desviación estándar serán los que se reporten para ser estudiados por el personal administrativo.

También es posible marcar factores de consumo excedidos. Por medio de las gráficas, pueden identificarse las fechas de cada trayectoria consultada en la cual el factor de consumo excede el límite superior. Teniendo en esta fecha, la matrícula de la aeronave y la ruta volada, se puede conocer qué piloto efectuó este vuelo. 


\section{Control de operaciones}

En la operación diaria de cualquier aerolínea normalmente existen retrasos por motivos técnicos, operacionales o incontrolables como el mal tiempo, el cierre temporal de aeropuertos, entre otros.

El personal de control vuelos debe realizar constantemente reprogramaciones sobre la marcha si ocurre alguna eventualidad. Cuando no se cuenta con una herramienta tecnológica como un software, se hace necesario hacer manualmente dicha reprogramación, no sólo de itinerarios sino también de aeronaves y tripulantes. Ello toma bastante tiempo y puede causar que la operación se retrase aún más y sea poco efectiva.

El software brinda al usuario, en primera instancia, una visualización panorámica de toda la operación del día, lo cual facilita la reprogramación de rutas, aeronaves y tripulantes en cuanto ofrece información diaria de la operación. Adicionalmente, puede ingresarse al sistema información como número de pasajeros transportados y horas reales de despegue y aterrizaje de las aeronaves para que las demás dependencias y bases de la compañía se encuentren también informados. De esta manera, el personal de control vuelos tendrá una disminución en la carga laboral, lo cual ayuda a que su labor sea más eficiente y efectiva.

El módulo de control de operaciones realiza las siguientes tareas: obtener información de la programación diaria para tener un estado de inicio; modificar información de la operación diaria de acuerdo a las circunstancias específicas del día; asignar rutas a la aeronave; adicionar una nueva aeronave a la operación y el itinerario de ésta (si es necesario); asignar tripulantes a rutas; calcular horas de vuelo acumuladas para el tripulante; identificar tripulante no apto para la operación diaria si este incumple la normatividad aeronáutica; registrar el número de pasajeros; registrar la hora de rodaje de la aeronave; registrar la hora de despegue de la aeronave; registrar información del retraso de la aeronave; registrar la hora de parqueo de la aeronave; registrar la hora de aterrizaje de la aeronave. Asimismo, puede registrar información adicional de cada vuelo como número de sillas ofrecidas, cantidad de carga ofrecida, cantidad de carga a bordo, cantidad de equipaje a bordo, combustible adicionado antes del despegue, cantidad de combustible al despegue, cantidad de combustible al aterrizar y correo a bordo.

La problemática actual de las empresas secundarias de transporte público regular en crecimiento es la necesidad de implementar una tecnología para mejorar sus procesos que se acomode a su presupuesto. Un software hecho a la medida se enfoca en los problemas específicos que existen al interior de cada empresa y sólo incluye los módulos que ésta exija, a diferencia de muchos programas existentes en el mercado que ofrecen un paquete de servicios que no se acomodan a las necesidades específicas de cada empresa y cuestan mucho dinero.

Al hacer el estudio de requisitos del sistema, se notó que cada empresa maneja sus procesos de acuerdo a ciertas políticas internas. Por lo anterior, se hace necesario un análisis profundo del área a tratar para ofrecer soluciones a través de procesos más prácticos y eficaces que hagan uso de herramientas tecnológicas como el software empresarial.

Las diferentes áreas de una empresa necesitan funcionar de manera sincronizada, en especial cuando se trata de empresas de aviación en lo referente al área de operaciones y mantenimiento. Si no hay un armónico funcionamiento de estas áreas, la operación de una aerolínea puede llegar a colapsar de tal manera que sus vuelos se retrasen, lo que acarrea multas para la compañía y pérdidas gigantescas. Para que exista un funcionamiento de las áreas simultáneo y correcto, es necesario (entre otras cosas) que exista una buena comunicación entre éstas, por lo cual se planteó una solución a través del programa SIO que crea una interfaz para que, por ejemplo, el usuario de mantenimiento pueda separar la aeronave para mantenimiento; así, control vuelos puede estar enterado de la disponibilidad de las aeronaves, al igual que el departamento de mantenimiento se entera si los aviones están en normal operación o hay problemas técnicos a solucionar.

Para una empresa de aviación comercial de transporte de pasajeros es de vital importancia que las dependencias que estén en contacto directo con el pasajero y estén enteradas de la operación y cumplimiento de la flota, de manera tal que puedan dar información oportuna al cliente. Sin el uso de herramientas tecnológicas como software e internet, se hace muy dispendioso para el personal de control vuelos notificar por radio o por teléfono a las dependencias el estado de los vuelos, máxime si la empresa tiene un número amplio de destinos. Al hacer uso de programas con aplicación web, todas las personas pueden estar enteradas de la operación en tiempo real sin tener que estar llamando a control vuelos, lo cual agilizaría la labor del personal de este departamento.

Al estudiar los requisitos del proceso de operaciones se lograron definir cinco módulos, a saber:

- Programación mensual de tripulantes.

- Programación diaria de aeronaves. 
CIENCIA Y PODER AÉREO | ISSN 1909-7050 | E-ISSN 2389-9468 | Vol. 12 | Ene - Dic 2017 | Escuela de Postgrados de la Fuerza Aérea Colombiana | pp $210-219$

- Programación diaria de tripulantes.

- Operación diaria.

- Estadísticas.

Estos cinco módulos abarcan todo el proceso de operaciones; sin embargo, para levantar todos los requisitos de estos módulos se hace necesario entrar al corazón del proceso y preguntar a cada persona que interviene directa o indirectamente cómo, cuándo, dónde y de qué forma interviene en el proceso. De no hacerlo, puede incurrirse en errores que luego de desarrollado el programa son muy complicados de solucionar en cuanto la labor de desarrollo de un software es dispendiosa y exigente.

La programación de tripulaciones en una empresa de aviación es de vital importancia. Realizar esta actividad de manera incorrecta podría acarrear multas y sanciones graves para una aerolínea, ya que a la hora de programar dichas tripulaciones, deben tenerse en cuenta requisitos de tiempo para los pilotos que son estipulados por la autoridad aeronáutica. Cuando una aerolínea maneja más de cincuenta tripulaciones y a una sola persona le corresponde verificar información de todos los pilotos, como los tiempos de servicio, vuelo, descanso y número de aterrizajes, es muy normal que ocurran errores por omisión. Por este motivo, el uso de un software de operaciones se vuelve indispensable, más en tiempos de crecimiento vertiginoso del sector.

Todas las industrias y empresas actuales acuden cada vez más al uso de la tecnología para mejorar sus procesos; la industria del transporte aéreo es una de ellas. Mediante el uso de los sistemas de información, pueden ofrecerse soluciones eficaces a problemas del día a día que ocurren en la aviación, con lo cual se ayuda al crecimiento sostenible de esta industria.

El crecimiento de la aviación mundial seguirá incrementandose inevitablemente, al igual que los inconvenientes del día a día. Asimismo, la demanda de ingenieros que ofrezcan soluciones oportunas a los problemas se hace cada vez más grande; por ello, es deber del ingeniero aeronáutico buscar soluciones a dichos problemas para seguir haciendo de la aviación el medio de transporte más seguro del mundo.

\section{RESULTADOS Y ANÁLISIS}

Al realizar la implementación del sistema SIO en la organización, se esperan los siguientes resultados en cada uno de los módulos:

\section{Módulo Programación Mensual:}

Reducción del tiempo en la elaboración de la programación mensual de tripulantes en cuanto el sistema ofrece una propuesta de programación automática que tiene en cuenta los tiempos de vuelo, servicio, vencimiento de entrenamientos, simulador, chequeos. Adicionalmente, al generar la programación mensual, el programa brinda la posibilidad de enviar una notificación por correo electrónico a todos los pilotos para que estén enterados de su programación para el mes.

Por otra parte, el SIO genera alertas cada mes al programador respecto a los vencimientos de cualquier novedad técnica de los pilotos sobre simulador, chequeo o recurrente.

\section{Módulo de Programación Diaria:}

El programa ofrece una propuesta automática de asignación de itinerarios a aeronaves con sus respectivos tripulantes. Valida que los itinerarios correspondan al tipo de aeronave, así como que la aeronave se encuentre disponible para vuelo y que la tripulación cumpla con los diferentes requisitos.

Una vez se genera la propuesta, el programador puede modificarla de acuerdo a sus necesidades específicas: puede eliminar un itinerario a una aeronave y asignarle otro de la lista de itinerarios cargados en la base de datos. También puede agregar o eliminar rutas del itinerario o modificar su horario. Si hay inconsistencias en las modificaciones que realice el usuario, el sistema genera alertas que indican tales inconsistencias. De igual manera, puede modificar tripulaciones en cualquier ruta del día y asignarle novedades técnicas como chequeos de ruta. Hay una interfaz que permite al personal de mantenimiento separar una aeronave para un servicio de mantenimiento en cualquier momento del día, así el programador está informado de si dispone o no de la aeronave.

\section{Módulo de Operación Diaria:}

Como ya se mencionó, en la operación de cualquier aerolínea se presentan situaciones que retrasan los vuelos, por lo que el personal de control debe constantemente realizar reprogramaciones sobre la marcha si ocurre alguna eventualidad. Cuando no se cuenta con una herramienta tecnológica como software, se hace necesario hacer manualmente dicha reprogramación, no sólo de itinerarios sino también de aeronaves y tripulantes, lo cual toma bastante tiempo y puede causar que la operación se retrase aún más y sea poco efectiva.

La solución dada por el software consiste en una visualización panorámica de toda la operación del día, lo cual facilita la reprogramación de rutas, aeronaves y tripulantes. Ofrece información en tiempo real de la operación, y pue- 
de ingresarse al sistema información como número de pasajeros a bordo y horas reales de despegue y aterrizaje de las aeronaves para que las demás dependencias y bases de la compañía se encuentren también informados. De esta manera, el personal de control vuelos tendrá una disminución en la carga laboral, lo que ayuda a que su labor sea más eficiente y efectiva.

\section{Módulo de Estadísticas:}

El software SIO captura automáticamente datos de la operación y genera tablas y gráficos de interés, tales como número de pasajeros transportados por ruta, estadística de vuelos demorados y estadística de consumo de combustible.

\section{CONCLUSIONES}

Todas las industrias, en especial la aérea, acuden al uso de la tecnología para mejorar sus procesos, al usar los sistemas de información se pueden ofrecer soluciones eficaces a problemas que se presentan en el día a día, permitiendo el desarrollo sostenible de la empresa.

Sin embargo, las empresas en crecimiento tienen la necesidad de implementar tecnología que se acomoden a su presupuesto. Un software hecho a la medida se enfoca en los problemas reales que se encuentran al interior de cada organización y sólo incluye los módulos que esta exija. Ello marca una diferencia respecto a muchos programas existentes en el mercado que ofrecen un paquete de servicios que no se acomodan a las necesidades específicas de cada empresa y que conllevan costos muy elevados.

Las diferentes áreas de una empresa aérea deben trabajar de forma sincrónica, especialmente las áreas de operaciones y mantenimiento. Para que exista esta coordinación, es necesaria una buena comunicación que puede lograrse a través del programa SIO.

La programación de tripulaciones es de vital importancia. Hacerlo de la manera incorrecta podría acarrear multas y sanciones graves para una aerolínea, ya que a la hora de programar dichas tripulaciones se deben tener en cuenta muchos requisitos entre estos el tiempo de servicio para las tripulaciones mismos que son estipulados por la autoridad aeronáutica. En ese contexto, el uso de un software de operaciones se vuelve casi que indispensable para la prevención de errores.

Para una empresa de aviación comercial de transporte de pasajeros, es de vital importancia el contacto directo con el pasajero. Por ello, deben estar enterados de la operación y la condición real flota, para ofrecer información oportuna al cliente.
El software planteado en su versión inicial presenta cuatro módulos y pasara por una etapa de implementación para verificar su eficiencia en condiciones reales de operación así como iniciar el proceso de mejoramiento continuo del mismo.

\section{Referencias}

Aeronautica Civil . (2011). Reglamentos Aeronauticos Colombianos. Parte Cuarta. Colombia.

Aeronautica Civil. (2011). Reglamentos Aeronauticos Colombianos. Parte Segunda. Colombia.

Aeronautica Civil. (2011). Reglamentos Aeronauticos Colombianos . Parte Primera. Colombia.

Álvarez Lara, S. A., \& García Echeverry, J. P. (2010). DISEÑO DE SOFTWARE: PLANEACIÓN CONTROL PRODUCCIÓN AERONÁUTICO. Revista Educacion en Ingenieria, 171-178.

Arteaga, L. (Junio de 2004). gestiopolis. Obtenido de http://www. gestiopolis.com/canales2/gerencia/1/ddsluisart.htm\#masautor

Cohen, D., \& Asin, E. (2001). Sistemas de información para los negocios. Un enfoque de toma de decisiones. McGraw-Hill.

Olivera, M., Cabrera, P., Bermúdez , W., \& Hernández, A. (2011). EL IMPACTO DEL TRANSPORTE AÉREO EN LA ECONOMÍA COLOMBIANA Y LAS POLÍTICAS PÚBLICAS. Colombia: La Imprenta Editores S.A.

Seicotechnology. (1 de Agosto de 2014). http://www.seicotechnology.com. Recuperado el 01 de 03 de 2017, de http:// www.seicotechnology.com/pdf/icarus_plus.pdf

VolarTec. (3 de Febrero de 2017). http://www.seaburysolutions. com/wp-content/uploads/2016/06/Seabury-SolutionsAlkym.pdf. Obtenido de http://www.seaburysolutions. com: http://www.seaburysolutions.com/wp-content/ uploads/2016/06/Seabury-Solutions-Alkym.pdf 